Ferrata Storti Foundation

\title{
Combined inhibition of MDM2 and BCR-ABL1 tyrosine kinase targets chronic myeloid leukemia stem/progenitor cells in a murine model
}

Haematologica 2020

Volume 105(5):1274-1284

\section{Correspondence: \\ BING Z. CARTER \\ bicarter@mdanderson.org \\ MICHAEL ANDREEFF \\ mandreef@mdanderson.org \\ Received: February 11, 2019. \\ Accepted: July 26, 2019. \\ Pre-published: August 1, 2019.}

doi:10.3324/haematol.2019.219261

Check the online version for the most updated information on this article, online supplements, and information on authorship \& disclosures: www.haematologica.org/content/105/5/1274

(C)2020 Ferrata Storti Foundation

Material published in Haematologica is covered by copyright. All rights are reserved to the Ferrata Storti Foundation. Use of published material is allowed under the following terms and conditions:

https://creativecommons.org/licenses/by-nc/4.0/legalcode. Copies of published material are allowed for personal or internal use. Sharing published material for non-commercial purposes is subject to the following conditions:

https://creativecommons. orǵ/licenses/by-nc/4.0/leǵalcode, sect. 3. Reproducing and sharing published material for commercial purposes is not allowed without permission in writing from the publisher.

\author{
Bing Z. Carter, ${ }^{1}$ Po Yee Mak, ${ }^{1}$ Hong Mu,${ }^{1}$ Xiangmeng Wang, ${ }^{1}$ Wenjing Tao, \\ Duncan H. Mak, ${ }^{1}$ Elisha J. Dettman, ${ }^{2}$ Michael Cardone, ${ }^{2}$ Oleg Zernovak, ${ }^{3}$ \\ Takahiko Seki, ${ }^{3}$ and Michael Andreeff ${ }^{1}$
}

${ }^{1}$ Section of Molecular Hematology and Therapy, Department of Leukemia, The University of Texas MD Anderson Cancer Center, Houston, TX, USA; ${ }^{2}$ Eutropics, Cambridge, MA, USA and ${ }^{3}$ Daiichi Sankyo Co. Ltd., Oncology Laboratories, R\&D Division, 2-58, Hiromachi 1-Chrome, Shinagawa-ku, Tokyo, Japan

\section{ABSTRACT}

A lthough highly effective, BCR-ABL1 tyrosine kinase inhibitors do not target chronic myeloid leukemia (CML) stem cells. Most patients relapse upon tyrosine kinase inhibitor therapy cessation. We reported previously that combined BCR-ABL1 and BCL-2 inhibition synergistically targets CML stem/progenitor cells. p53 induces apoptosis mainly by modulating BCL-2 family proteins. Although infrequently mutated in CML, p53 is antagonized by $\mathrm{MDM} 2$, which is regulated by BCR-ABL1 signaling. We hypothesized that MDM2 inhibition could sensitize CML cells to tyrosine kinase inhibitors. Using an inducible transgenic Scl-tTa-BCR-ABL1 murine CML model, we found, by RT-PCR and CyTOF proteomics increased p53 signaling in CML bone marrow (BM) cells compared with controls in CD45 and linage-SCA- $1^{+} \mathrm{C}-\mathrm{KIT}{ }^{+}$populations. CML BM cells were more sensitive to exogenous $\mathrm{BH} 3$ peptides than controls. Combined inhibition of BCR-ABL1 with imatinib and MDM2 with DS-5272 increased NOXA level, markedly reduced leukemic linage-SCA- $1^{+} \mathrm{C}-\mathrm{KIT}^{+}$cells and hematopoiesis, decreased leukemia burden, significantly prolonged the survival of mice engrafted with $\mathrm{BM}$ cells from Scl-tTa-BCR-ABL1 mice, and significantly decreased CML stem cell frequency in secondary transplantations. Our results suggest that CML stem/progenitor cells have increased p53 signaling and a propensity for apoptosis. Combined MDM2 and BCR-ABL1 inhibition targets CML stem/progenitor cells and has the potential to improve cure rates for CML.

\section{Introduction}

Chronic myeloid leukemia (CML) originates from the $\mathrm{t}(9 ; 22)$ chromosomal translocation that results in the BCR-ABL1 fusion gene and constitutive activation of the BCR-ABL1 tyrosine kinase in hematopoietic stem cells. ${ }^{1.3} \mathrm{CML}$ stem cells are quiescent, ${ }^{4}$ yet can self-renew, proliferate, differentiate, and promote expansion of the myeloid lineage. The development of imatinib and other tyrosine kinase inhibitors (TKI) has made CML, once a deadly disease, highly manageable with a 10-year overall survival rate of over $90 \%$.

Although extremely effective in eliminating proliferating CML cells, TKI are inactive against quiescent CML stem cells, despite inhibition of BCR-ABL1 activity, ${ }^{5.7}$ and several clinical trials have demonstrated that approximately $50 \%$ of patients eventually relapse after ceasing TKI therapy ${ }^{8.11}$ Long-term treatment with TKI is expensive, and may lead to the development of inhibitor resistance, or intolerance to therapy. Furthermore, the persistence of CML stem cells contributes to the generation of new clones with additional acquired mutations, which can lead to progression to acute disease over time. Thus, eradicating CML stem cells is the ultimate goal in curing CML.

Numerous combinatorial strategies have been proposed pre-clinically and shown to be effective in eradicating CML stem cells..$^{12-16}$ Among them, concomitant targeting of 
anti-apoptotic BCL-2 proteins enhances TKI activity in $\mathrm{CML},{ }^{17-19}$ and we demonstrated that BCL-2 is a key survival factor of CML stem cells, and targeting BCL-2 with ABT199, combined with a TKI, enhanced eradication of CML stem cells. $^{20}$

Among its numerous tumor suppressor functions, p53 activates the expression of the pro-apoptotic BCL-2 proteins BAX, PUMA, NOXA, and BID triggering apoptosis. ${ }^{21-}$ ${ }^{23}$ Altered p53 and MYC transcriptional network in CML stem cells was recently reported, and targeting both p53 and MYC selectively eliminated CML stem cells. ${ }^{24}$ Activation of p53 by inhibition of SIRT1 or MDM2, in combination with TKI has been explored in CML. ${ }^{25,26}$ We reported that TKI in combination with the MDM2 inhibitor nutlin3a enhanced apoptosis induction in proliferating and quiescent blast crisis CML progenitor cells in vitro. ${ }^{27}$

The expression of p53 can be induced when cells are stressed, including those associated with oncogenic stimulation. Like other oncogenes, the hyper-proliferative signal from $B C R-A B L 1$ can activate p53 and induce cell cycle block and senescence to counterbalance oncogenic stimulation signals. This may also contribute to CML stem cell maintenance. However, the role of p53 signaling proteins in BCRABL1 oncogene-driven CML/CML stem cells and the response of CML stem cells to the combined MDM2 and BCR-ABL1 inhibition have not been fully investigated.

Using an inducible, stem cell promoter ( $\mathrm{Scl}$ )-driven transgenic CML murine model (Scl-tTa- $B C R-A B L 1$ mice), ${ }^{15,20,28,29}$ we here determine the expression of $\mathrm{p} 53$ and its signaling proteins in bone marrow (BM) cells and lineage-SCA- $1^{+} \mathrm{C}-$ $\mathrm{KIT}^{+}$(LSK) cells from CML and control mice, and in BM cells in CML mice treated with the MDM2 inhibitor DS5272, the TKI imatinib, or both, using novel CyTOF mass cytometry, which measures single-cell protein expression in phenotypically-defined cell populations. We also investigated the anti-leukemia activity of combined MDM2 and BCR-ABL1 inhibition in this model.

\section{Methods}

\section{Mouse model and cells}

Mouse experiments were performed in accordance with $\mathrm{MD}$ Anderson Cancer Center Animal Care and Use Committee approved protocols. Scl-tTa-BCR-ABL1 FVB/N mice ${ }^{28,29}$ were provided by Dr. R. Bhatia (University of Alabama at Birmingham, AL, USA). BM cells were collected from mice 3-4 weeks after tetracycline cessation (Tet-off) or from controls (Tet-on).

\section{Human cells}

Cells from newly diagnosed chronic phase CML (CML-CP) patients (Online Supplementary Table S1) and normal controls were obtained as described in the Online Supplementary Methods.

\section{Real-time polymerase chain reaction}

Real-time polymerase chain reaction (RT-PCR) was carried out as previously described ${ }^{20}$ using freshly isolated mouse or human BM cells. Primers are shown in Online Supplementary Table S2. The abundance of each transcript relative to that of Abl1 or ABL1 was calculated using the $2^{-\Delta \mathrm{Ct}}$ method, expressed as copies of each mRNA/1000 copies of Abl1 or ABL1.

\section{Western blot}

Western blot was performed as described previously. ${ }^{20}$ Antibodies against human p53 and BAX were purchased from Santa Cruz (Dallas, TX, USA).

\section{Mass cytometry}

Mouse BM cells were stained with metal-tagged antibodies for cell surface markers and intracellular proteins (Online Supplementary Table S3) and subjected to mass cytometry (CyTOF) analysis as previously described ${ }^{16,20,30}$ and as briefly described in the Online Supplementary Methods.

\section{Mitochondrial priming}

BH3 priming assay as previously described ${ }^{31}$ is described briefly in the Online Supplementary Methods.

\section{In vivo experiments}

$\mathrm{GFP}^{+} \mathrm{CML}$ cells from donor mice as previously described ${ }^{15,20}$ were injected $\left(0.6 \times 10^{6}\right.$ cells/mouse) into FVB/N recipient mice (The Jackson Laboratory) irradiated at 900 cGy. After CML developed, assessed by flow cytometry measurement of GR-1 (LY6G) cells, mice were treated daily (oral gavage) with imatinib $(100 \mathrm{mg} / \mathrm{kg}$; vehicle: acidified water, pH 5.0) for four weeks, DS-5272 (50 $\mathrm{mg} / \mathrm{kg}$; vehicle: $0.5 \% \mathrm{w} / \mathrm{v}$ methylcellulose 400 ) for two weeks (initiated two weeks after imatinib group), imatinib for two weeks and then plus DS-5272 for two additional weeks, or vehicle control (1:1 volume of each vehicle). Two sets of experiments were performed.

Experiment I: at the end of treatments, BM and spleen cells ( $\mathrm{n}=3$ 5/group) were collected and stained with a lineage cocktail and antibodies against SCA-1 (eBioscience, ThermoFisher Scientific), CKIT (CD117), CD34, FcrRII/III, GR-1 (LY6G), and MAC-1 (CD11b) (all from BioLegend, San Diego, CA, USA) to measure leukemia LSK, myeloid progenitors, and myeloid cells as previously described. ${ }^{20}$ Peripheral blood (PB) leukemia burden was measured by total and $\mathrm{GFP}^{+}$white blood cell (WBC) count $\left(\mathrm{CD} 45^{+}\right)$and total and $\mathrm{GFP}^{+}$neutrophils $\left(\mathrm{Ly} 6 \mathrm{G}^{+}\right)$using flow cytometry. Mouse survival was recorded.

Experiment II: BM cells were collected at the end of treatments for secondary transplantation as described previously. ${ }^{20}$ At 16 weeks, PB engraftment was determined, and the frequency of leukemia long-term hematopoietic stem cells (LT-HSC) was calculated. Green fluorescent protein (GFP) positivity and BCR-ABL1 RNA levels in BM cells from mice that received $0.25 \times 10^{6}$ cells/mouse were determined.

\section{Statistical analysis}

Results are expressed as mean \pm standard error of the mean. $P<0.05$ was considered statistically significant (two-sided Student $t$ test). Correlation coefficient was determined by Pearson correlation analysis. Mouse survival was estimated by the Kaplan-Meier method and data were analyzed using the log-rank test. LT-HSC frequency was calculated using the Extreme Limiting Dilution Analysis software program (http://bioinf.wehi.edu. au/software/elda/). ${ }^{32}$

\section{Results}

BCR-ABL1 oncogene increases the expression of p53 and p53 targets

To determine p53 expression and p53 signaling in hematopoietic cells of CML mice and compare with those of controls, we collected BM cells from Tet-off CML and Tet-on control Scl-tTa-BCR-ABL1 FVB/N mice, and determined the RNA levels of p53 and its target genes by RTPCR. We found that BCR-ABL1 induction significantly increased Trp53 (p53) RNA and that of its target genes including Bax, Pmaip1 (Noxa), Mdm2, and Cdkn1a (p21) (Figure $1 \mathrm{~A})(P<0.001$ for all genes analyzed), supporting oncogenic induction of p53. 
A
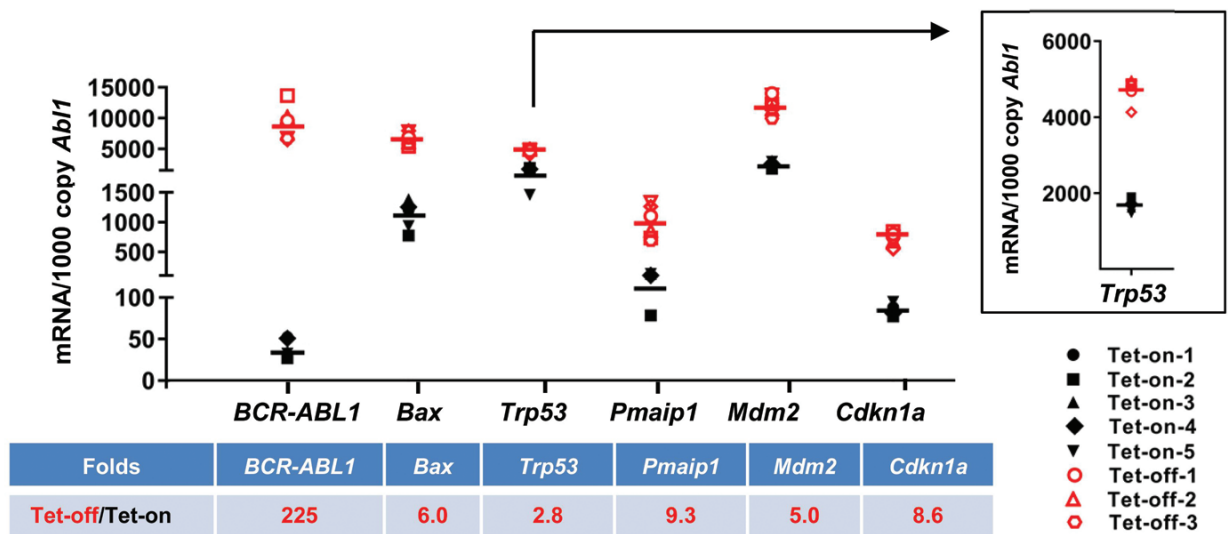

- Tet-on-1

- Tet-on-2

- Tet-on-3

Tet-on-4

v Tet-on-5

- Tet-off-1

Tet-off-2

$\triangle$ Tet-off-2

Tet-off-3

$\nabla$ Tet-off-5

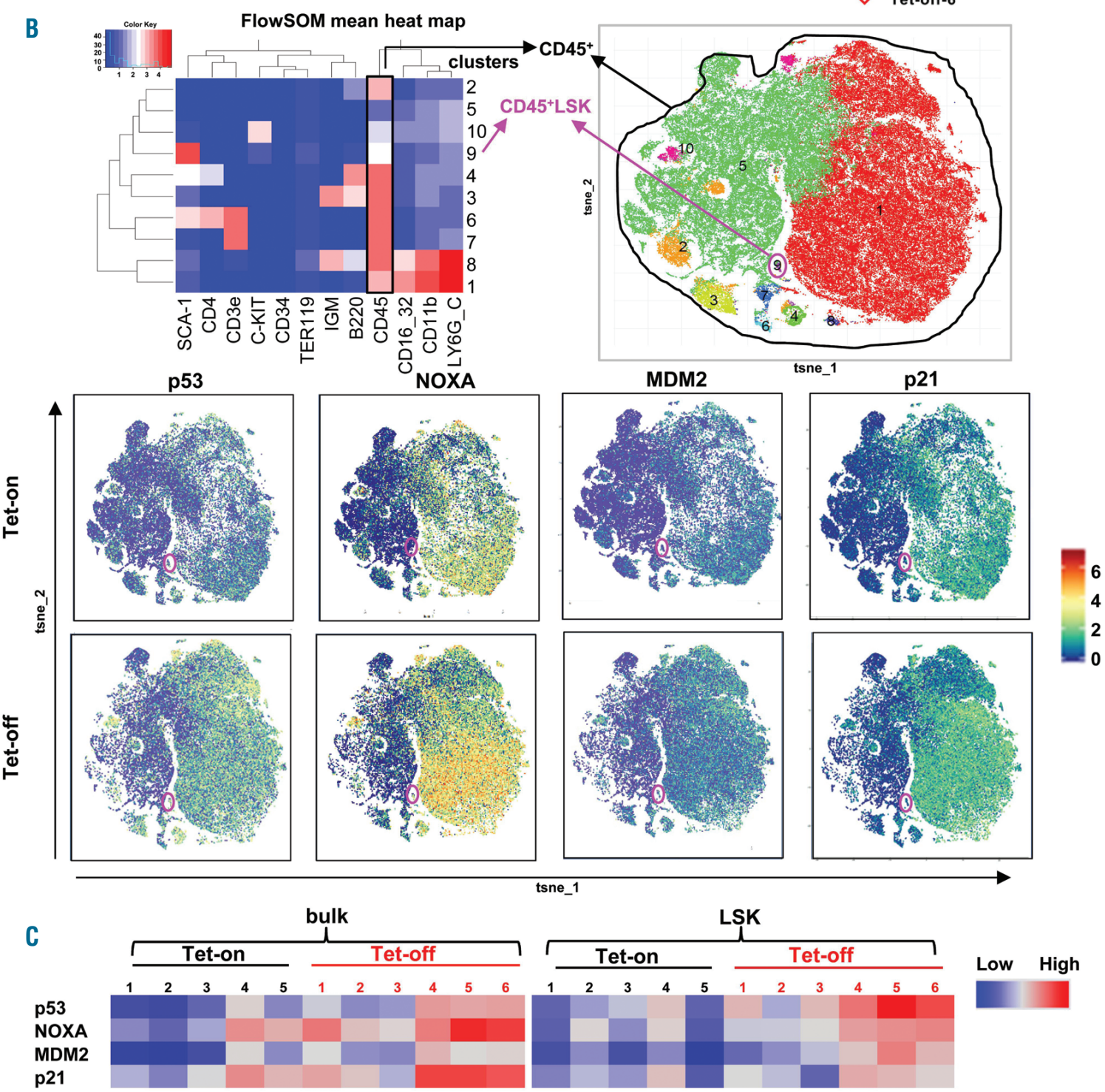

Figure 1. Expression of $\mathrm{p} 53$ and target genes in Tet-off chronic myeloid leukemia (CML) and Tet-on control mice. (A) RNA levels of Trp53 (also shown in the insert) and its target genes in Tet-off CML and Tet-on control mouse bone marrow (BM) cells, determined by Taq-Man real-time polymerase chain reaction (RT-PCR). (B) Heat map and cell subset clusters by RPhenoGraph based on mouse cell surface markers (upper) and protein expression of p53 and p53 targets in BM cells of Tet-off and Tet-on mice, shown by RPhenoGraph (constructed from all the samples in each group) (lower panel) determined by CyTOF mass cytometry analysis. (C) Heat map showing protein expression of p53 and target genes in individual Tet-off CML and Tet-on control mouse BM CD45 $5^{+}$(bulk) and lineage-SCA-1 $1^{+} \mathrm{C}-\mathrm{KIT}^{+}$(LSK) cells as determined by CyTOF mass cytometry, quantified by FlowJo software, and expressed in ArcSinh. BM cells were collected from mice 3-4 weeks after tetracycline cessation (Tet-off) $(n=6)$ or from age-matched controls (Tet-on) $(n=5)$. 
We next stained BM cells from Tet-off and Tet-on ScltTa-BCR-ABL1 FVB/N mice with a panel of metal-tagged antibodies for cell surface and intracellular proteins (Online Supplementary Table S3) and performed CyTOF mass cytometry analysis. Following Cytofkit unsupervised subset detection based on RPhenoGraph clustering algorithms, expression of various proteins was determined in bulk $\mathrm{CD}_{45^{+}}$and LSK (cluster 9, pink circle) cells (see Figure $1 B$ ). We found increased levels of p53 and its targets, including NOXA, MDM2, and p21 in CD45 ${ }^{+}$and LSK cells from Tet-off BM cells compared with their Tet-on counterparts, as displayed by RPhenoGraph (Figure 1B) as well as in a heat map showing the expression of each protein in each individual mouse (Figure 1C). We previously reported that BAX, another target of p53, was also higher in BM CD $45^{+}$and LSK cells from Tet-off CML mice compared to that in control mice. ${ }^{20}$
To demonstrate that increased expression of p53 and its target proteins also occurs in newly-diagnosed CML-CP patients, we first performed RT-PCR using RNA isolated from fresh BM samples of patients $(n=5)$ (Online Supplementary Table S1) and normal controls $(\mathrm{n}=6)$. Although there were high individual variations, overall BM cells from CML patients expressed higher levels of RNA representing p53 signaling proteins compared with those from normal controls, with statistically significantly higher $B A X(P=0.009)$ and markedly higher PMAIP1 (NOXA) $(P=0.06)$ (Figure 2A).

We next isolated CD34+ cells from fresh $\mathrm{BM}$ or $\mathrm{PB}$ samples of newly-diagnosed CML patients ( $\mathrm{n}=7$ ) (Online Supplementary Table S1) and fresh BM samples from normal controls $(n=5)$ and obtained sufficient material for determining p53 and BAX protein levels by western blot analysis. We found that CD34+ cells from CML-CP patient
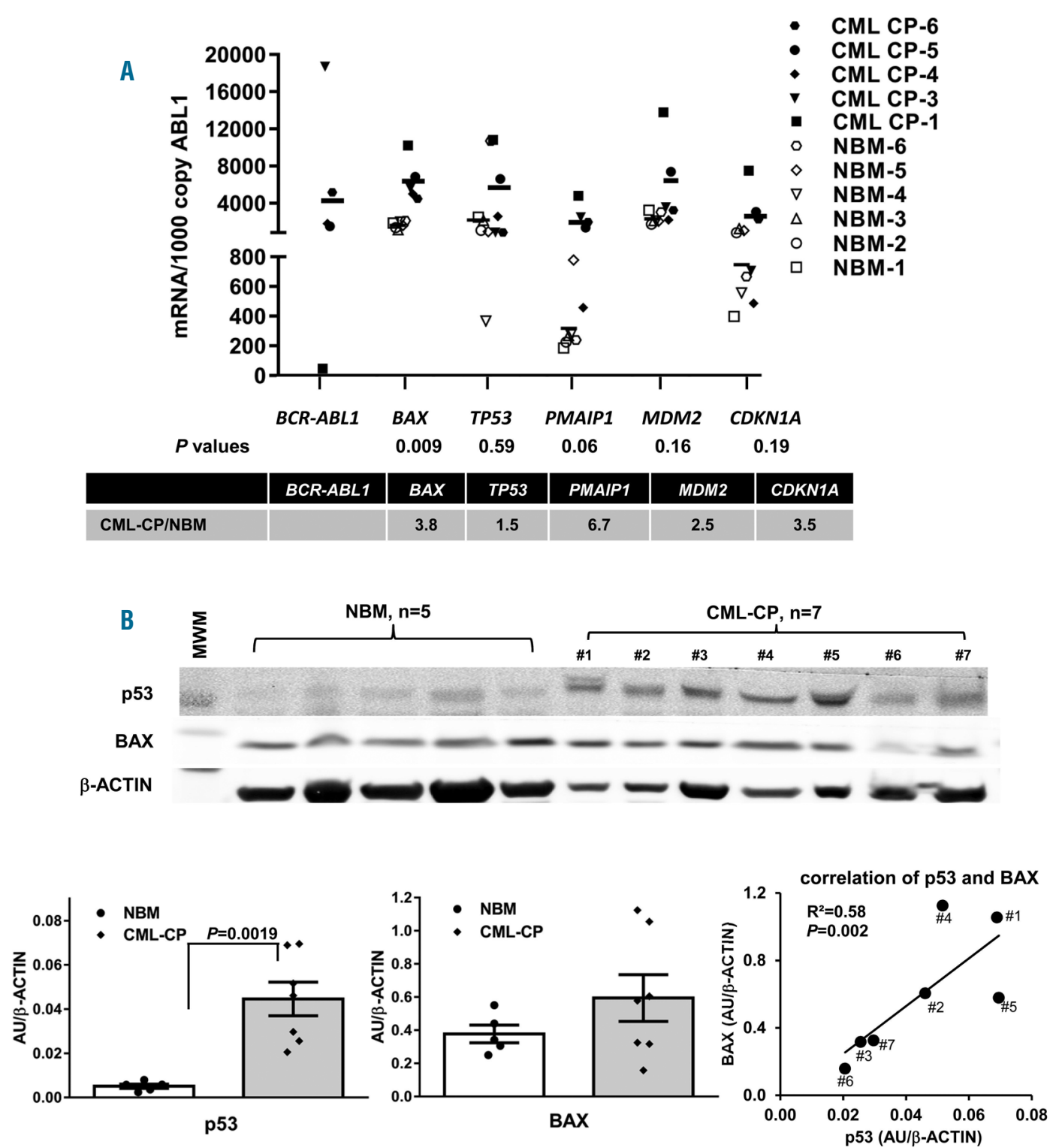

Figure 2. Expression of $\mathrm{p} 53$ and target genes in samples from patients with chronic phase chronic myeloid leukemia (CML)-CP and normal bone marrow (BM) controls (NBM). RNA and protein lysates were prepared from freshly collected samples and CML samples were obtained from untreated newly diagnosed CML-CP patients. (A) RNA levels of TP53 and its target genes in CML patient samples $(n=5)$ and NBM controls $(n=6)$, determined by Taq-Man real-time polymerase chain reaction. (B) p53 and BAX protein levels in CD34 ${ }^{+}$cells of NBM $(n=5)$ or of untreated newly diagnosed CML-CP patients $(n=7)$ were determined by western blot analysis and the correlation of the two proteins in patient samples was shown. MWM: molecular weight markers. Error bars indicate standard error of the mean. 
samples expressed statistically significantly higher p53 $(P=0.019)$ and, although non-significant, higher BAX protein levels, and that p53 and BAX levels in the CML-CP samples were highly correlated $\left(\mathrm{R}^{2}=0.58, P=0.002\right)$ (Figure $2 \mathrm{~B})$. Note among the seven CML-CP samples tested, sample \#6, that had the lowest levels of p53 and BAX, was a $\mathrm{PB}$ sample. All of the other samples were derived from $\mathrm{BM}$. All the normal controls were derived from BM.
Bone marrow lineage-SCA-1 ${ }^{+} \mathrm{C}-\mathrm{KIT}^{+}$cells from chronic myeloid leukemia mice are sensitive to $\mathrm{BH} 3$ peptide-induced apoptosis

Induction of apoptosis is one of the major functions of p53, which occurs primarily through transcriptional activation of pro-apoptotic BCL-2 family proteins such as BAX and NOXA. Our previous study of the overexpression of BCL- 2 proteins ${ }^{20}$ and the present assessment of

A

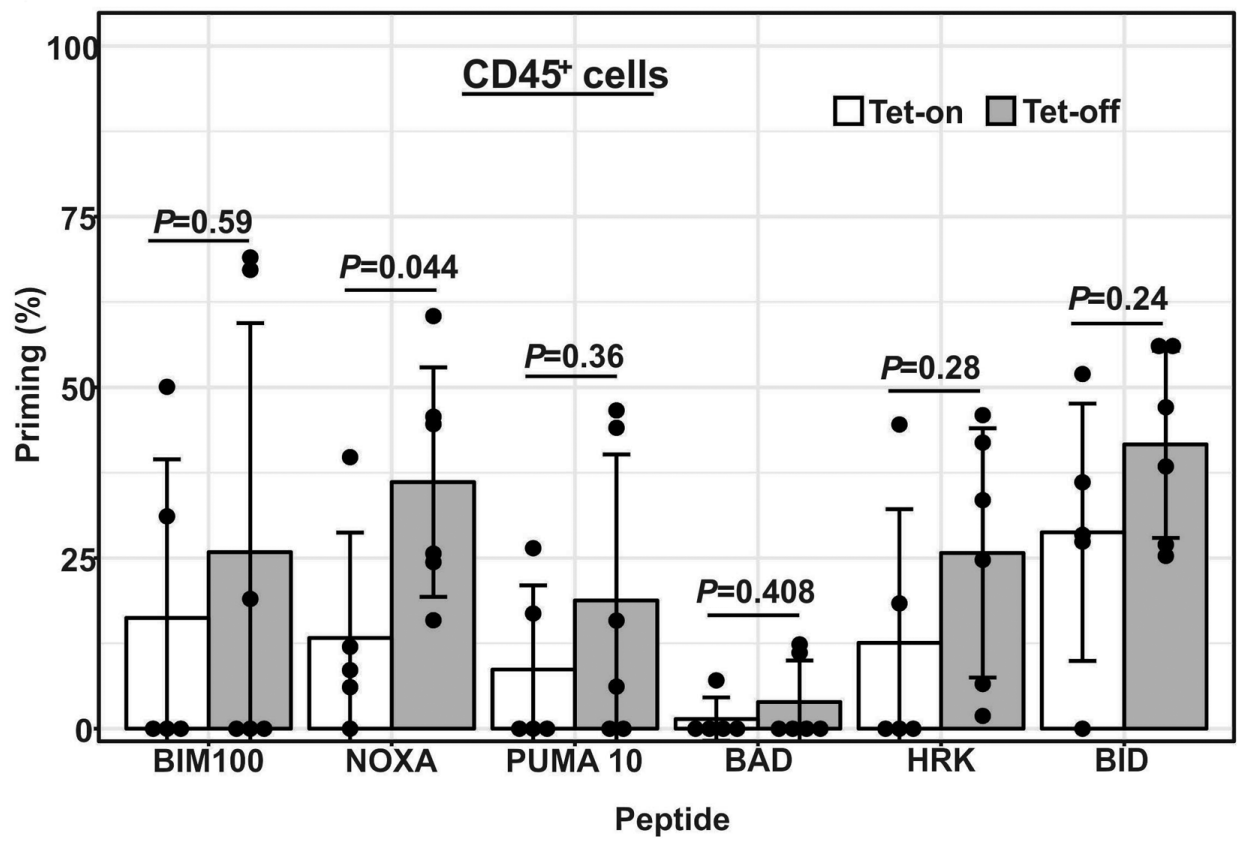

B

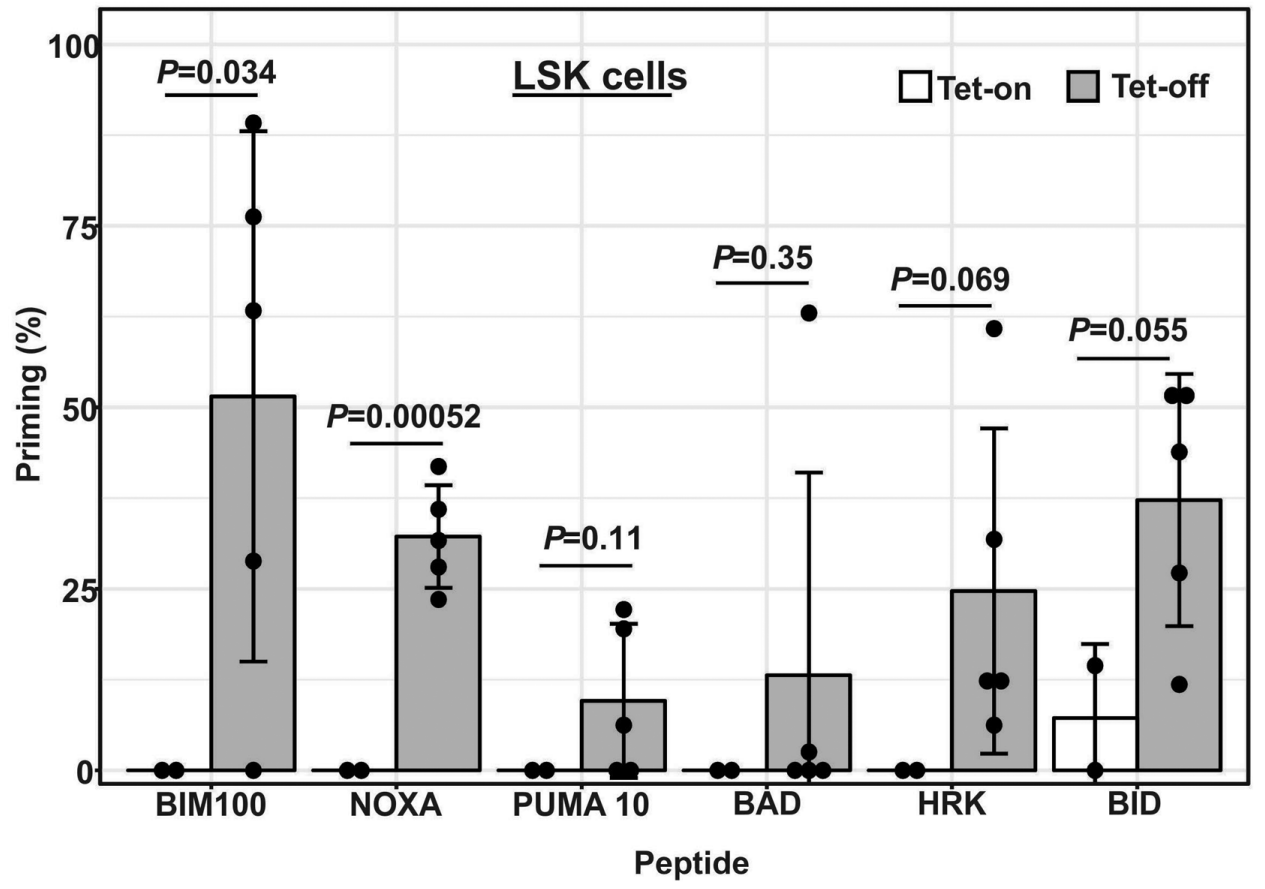

Figure 3. Priming analysis of bone marrow (BM) cells from Tet-off chronic myeloid leukemia (CML) and Tet-on control mice to BH3 peptides. BM cells were treated with various BH3 peptides (PUMA, $10 \mu \mathrm{M}$; all others, $100 \mu \mathrm{M}$ ) for 2 hours and 15 minutes. CD45+ or lineage-SCA-1+C-KIT+ $(\mathrm{LSK})$ cells were analyzed using flow cytometry after the cells were stained with JC-1 and priming was calculated for each peptide. Error bars indicate standard error of the mean. BM cells were collected from mice 3-4 weeks after tetracycline cessation (Tet-off) $(n=6)$ or from age-matched controls (Tet-on) $(n=5)$. (A) Results of CD45 cells $(n=6$ for Tet-off and $n=5$ for Teton). (B) Results of LSK cells ( $n=5$ for Tet-off and $n=2$ for Tet-on). 
increased p53 signaling in the BM of CML mice suggested that CML cells are protected from cell death, but possibly could still have a propensity for p53-mediated apoptosis. Consequently, they could be more sensitive to BH3 peptides than normal controls. To test this, we treated BM cells from Tet-off CML $(n=6)$ and Tet-on control $(n=5)$ mice with various $\mathrm{BH} 3$ peptides in order to assess the pool of sequestered pro-apoptotic, BH3-only proteins (priming) in $\mathrm{CD}_{4} 5^{+}$and $\mathrm{CD} 45^{+} \mathrm{LSK}$ cells. Owing to the limited number of cells available in the LSK population, priming results were calculated for only those samples in which sufficient LSK cells ( $>10$ cells) were measured ( $n=5$ for Tet-off and $\mathrm{n}=2$ for Tet-on).

Although the variations were large, (likely due, in part, to limited cell numbers in some samples), CML mouse BM $\mathrm{CD} 45^{+}$cells tended to be more sensitive to the BH3 pep-

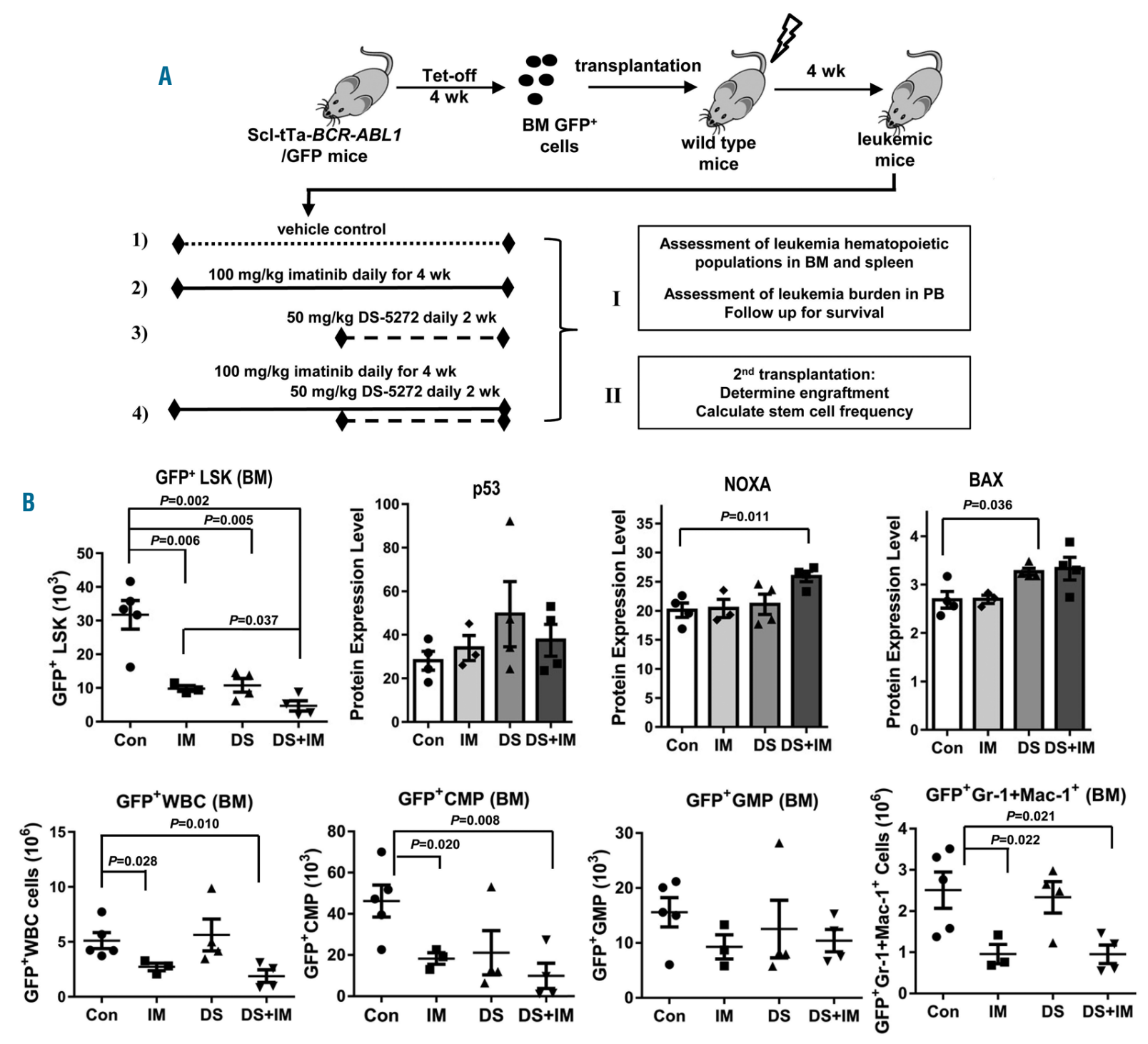

C

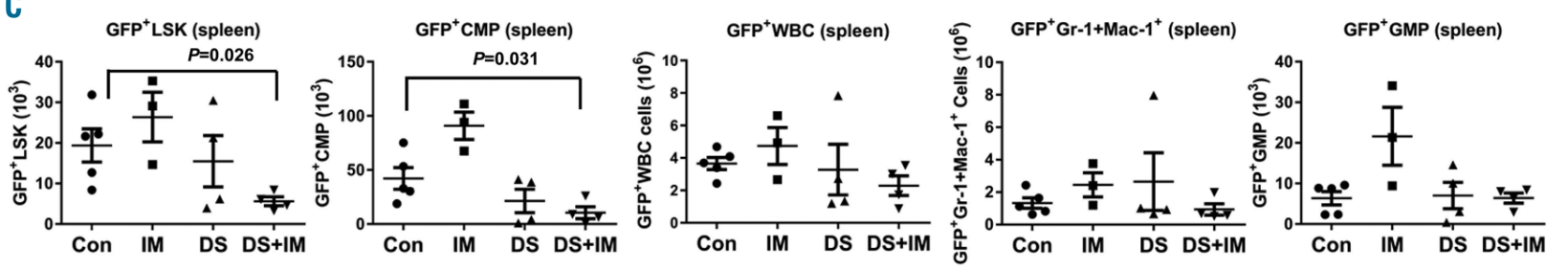

Figure 4. Effects of combined activation of $\mathrm{p} 53$ by MDM2 inhibition and inhibition of BCR-ABL1 by imatinib in vivo. (A) Experimental scheme. (B) The combination of the MDM2 inhibitor DS-5272 (DS) and imatinib (IM) significantly decreases chronic myeloid leukemia (CML) lineage-SCA-1+C-KIT ${ }^{+}(\mathrm{LSK})$ frequency, increases p53 signaling in CML LSK cells, and reduces leukemia cells in various cell subsets in mouse bone marrow (BM). (C) The combination of DS and IM significantly decreases CML LSK frequency and reduces leukemia cells in various cell subsets in the mouse spleen. The analysis was carried out in cells collected at the end of the treatments. Con: control. (B and C) N=3, 4, and 4 for IM, DS, and IM+DS treatment groups; respectively. In the control group, $n=5$ for measuring CML cell numbers in various populations, and $n=4$ for determining protein levels by CyTOF mass cytometry. 
to limited cell numbers in some samples), CML mouse BM $\mathrm{CD} 45^{+}$cells tended to be more sensitive to the $\mathrm{BH} 3$ peptides tested, but were statistically significantly more sensitive than cells from control mice to the NOXA peptide $(P=0.044)$ (Figure $3 \mathrm{~A})$. CML mouse BM LSK cells overall were statistically significantly more sensitive to $\mathrm{BH}$ peptides, especially to BIM $(P=0.034)$ and NOXA $(P=0.00052)$, than were controls (Figure $3 \mathrm{~B}$ ). These results indicated that CML cells, and stem/progenitor cells, were more sensitive to BH3 peptide-induced apoptosis than controls.
Combined activation of p53 and inhibition of BCR-ABL1 demonstrates strong anti-leukemia activity and inhibits leukemic lineage-SCA-1+C-KIT'cells in chronic myeloid leukemia mice

Bone marrow cells from Tet-off Scl-tTa-BCR-ABL1/GFP mice were injected into irradiated recipient $\mathrm{FVB} / \mathrm{N}$ mice. After confirming the development of neutrophilic leukocytosis in PB (4 weeks after cell injection), mice were untreated, or treated with imatinib, DS-5272, or the combination, following the scheme shown in Figure 4A. At the
A End of treatment
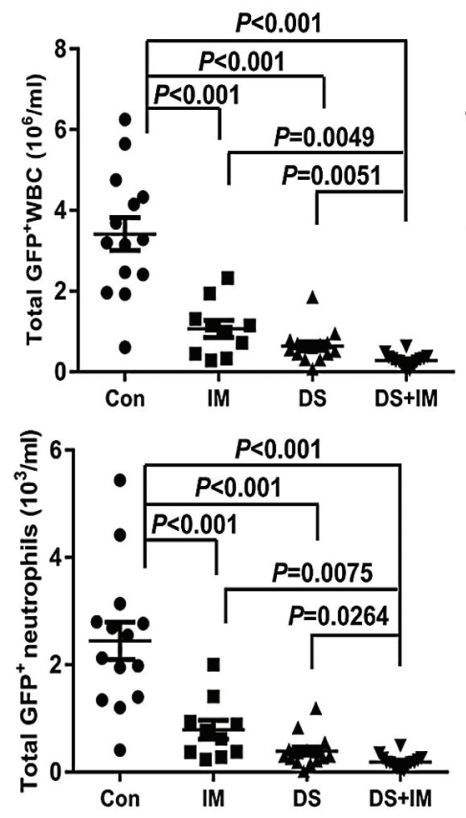

B $\underline{7 \text { wk after treatment }}$
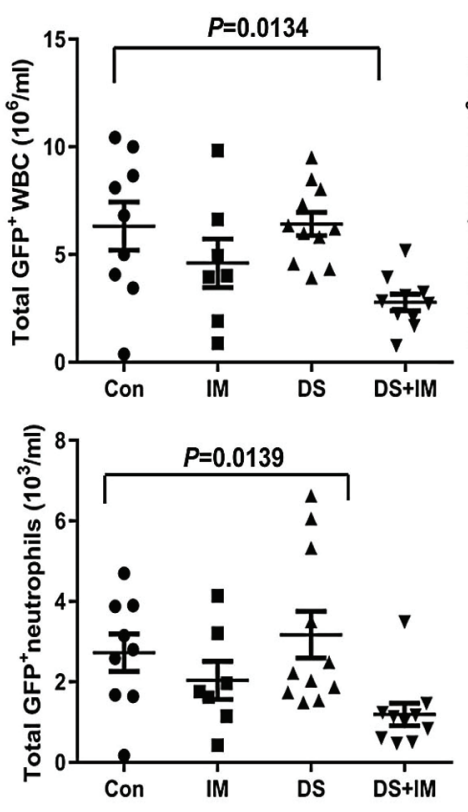

11 wk after treatment
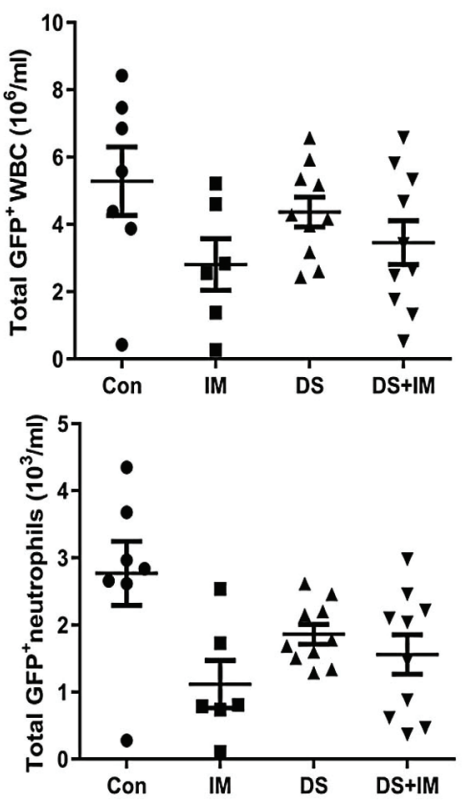

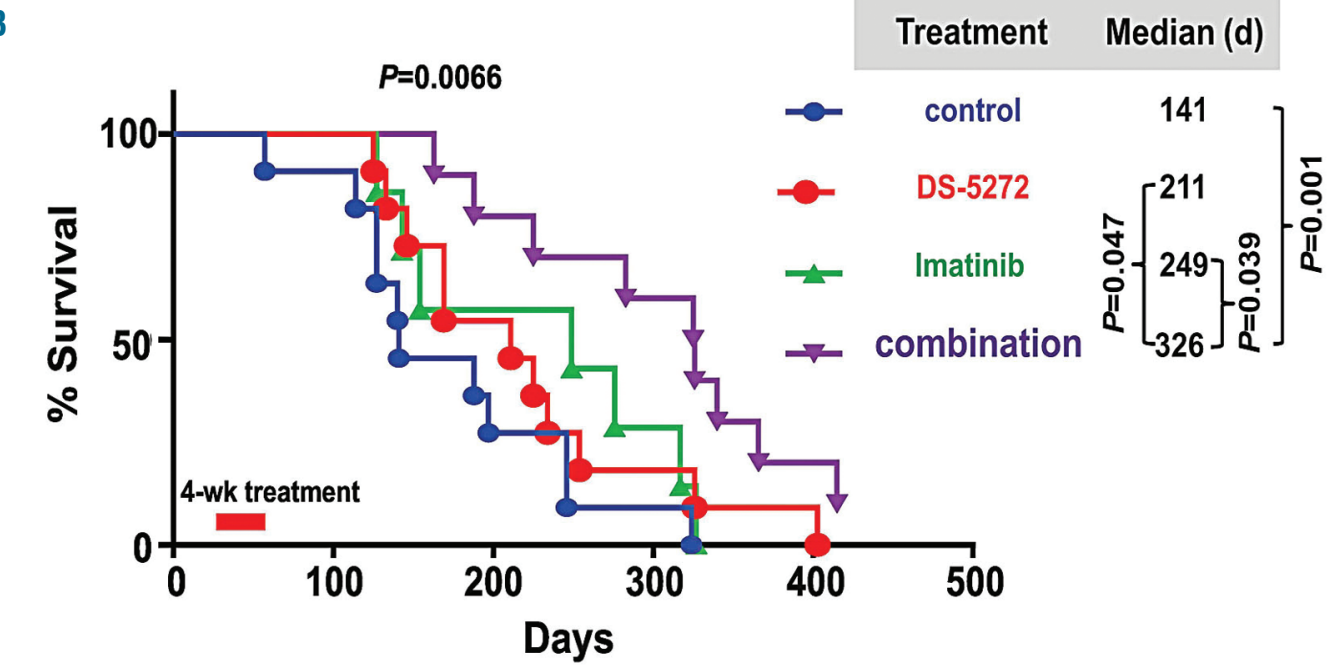

Figure 5. Anti-leukemia activity of combined activation of p53 by MDM2 inhibition and inhibition of BCR-ABL1 by imatinib in a mouse model of chronic myeloid leukemia (CML). (A) Leukemia burden in each treatment group at the end of treatments and 7 and 11 weeks (wk) after treatments was assessed using flow cytometry measuring total GFP ${ }^{+}$white blood cell (WBC) count and neutrophils in mouse peripheral blood (PB). Con: control; IM: imatinib; DS: DS-5272. (B) Kaplan-Meier curves for overall survival. Each dot/mark represents the result from an individual mouse; d: days. 
end of the 4-week treatments, BM and spleen cells were collected ( $n=3-5$ from each group) and leukemia hematopoiesis was analyzed. Flow cytometry analysis showed that the number of GFP+LSK cells was significantly reduced in the $\mathrm{BM}$ in all treatment groups compared to controls (Figure 4B). The combination was the most effective $\left(P=0.002\right.$ vs. control; $\mathrm{GFP}^{+} \mathrm{LSK}$ cell numbers in combination was $14.9 \%$ of that in control group) and was statistically significantly better than imatinib alone $(P=0.037)$ (Figure $4 \mathrm{~B})$. The combination also statistically significantly decreased the number of GFP-LSK cells $(P=0.026$ vs. control), but to a much lesser degree, and GFP-LSK cells in the combination treated group was $38.5 \%$ of that in the con- trol, suggesting that a therapeutic window exists for the respective treatments (Online Supplementary Figure S1A).

We determined the levels of p53 and its target proteins in the LSK cell population by CyTOF mass cytometry and observed some increases in these proteins, particularly significant increases of NOXA in the combination group and BAX in the DS-5272-treated group (Figure 4B). BAX level was also increased in the combination group, but did not reach statistical significance (Figure $4 \mathrm{~B}$ ). Imatinib and the combination also statistically significantly decreased the number of $\mathrm{GFP}^{+} \mathrm{WBC}$, common myeloid progenitor $(\mathrm{CMP})$ cells, and mature myeloid cells, and granulocytemonocyte progenitor (GMP) cells were not significantly
A

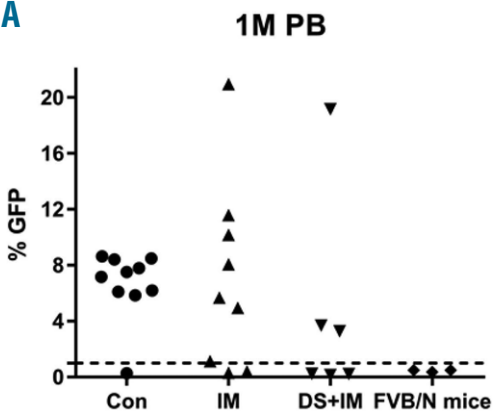

0.5M PB

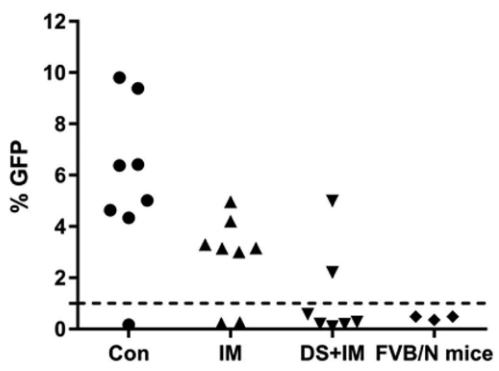

0.25M PB

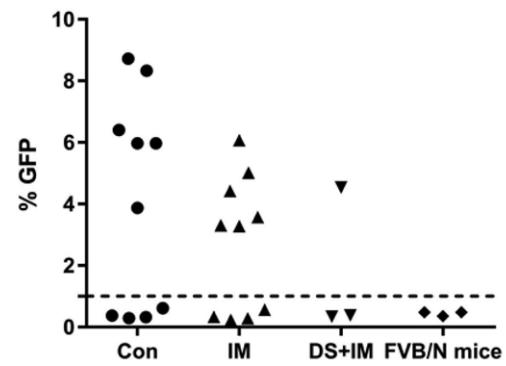

\begin{tabular}{|c|c|c|c|}
\hline & \multicolumn{3}{|c|}{ Engrafted/tested (PB GFP+>1\%), $P=0.0039$} \\
\hline cell dose & no treatment & imatinib & DS-5272 + imatinib \\
\hline $1 \times 10^{6}$ & $9 / 10$ & $7 / 9$ & $3 / 6$ \\
\hline $0.5 \times 10^{6}$ & $7 / 8$ & $6 / 8$ & $2 / 7$ \\
\hline $0.25 \times 10^{6}$ & $6 / 10$ & $6 / 10$ & $1 / 3$ \\
\hline LT-HSC frequency & 31.9E-07 & 23.3E-07 & 7.6E-07 \\
\hline
\end{tabular}

B

$0.25 M B M$
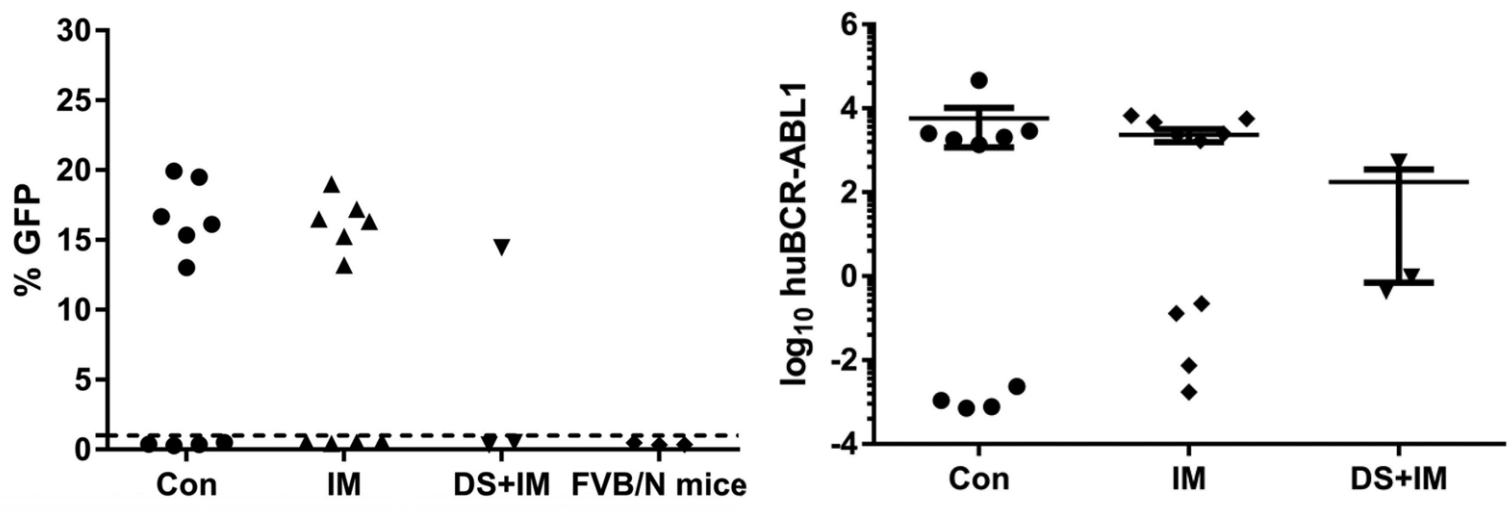

Figure 6. Effects of combined inhibition of MDM2 and BCR-ABL1 on chronic myeloid leukemia (CML) stem cells in vivo after secondary transplantation. (A) Green fluorescent protein (GFP) positivity (top panels) and numbers of engrafted versus total transplanted mice and leukemia long-term hematopoietic stem cell (LT-HSC) frequency (lower panel) are shown at 16 weeks after secondary transplantation in peripheral blood (PB) of mice injected with various cell dilutions for each treatment group. (B) GFP positivity at 16 weeks after secondary transplantation in bone marrow (BM) of mice injected with $0.25 \times 10^{6}$ cells/mouse in each treatment group, as well as BM BCR-ABL1 RNA levels in these mice. Each dot/mark represents the result from an individual mouse. M: one million cells. 
affected by the treatments (Figure 4B) at this time point.

Although we did not observe decreased spleen leukemic hematopoiesis by imatinib (but rather some increase) or DS-5272, the combination statistically significantly reduced leukemia GFP'LSK (Figure 4C), while neither single agent alone, nor their combination, statistically significantly altered the number of GFP-LSK in mouse spleen (Online Supplementary Figure S1B). In addition, the combination also statistically significantly decreased $\mathrm{GFP}^{+} \mathrm{CMP}$ cells in spleen and tended to reduce leukemia WBC and mature myeloid cells (Figure 4C). Again, the treatments did not affect GMP cells in the spleen.

Mice that received DS-5272 were treated for only two weeks during the second half of the treatment (Figure 4A). Although DS-5272 by itself did not statistically significantly decrease CML cells in several cell populations, it greatly enhanced the activity of imatinib, especially in suppressing GFP+LSK cells, both in BM and spleen.

To further assess the anti-leukemia activity of each agent and the combination, we determined circulating leukemia at the end of and at 7 and 11 weeks after treatments by flow cytometry measurement of $\mathrm{GFP}^{+}$total $\mathrm{WBC}$ and $\mathrm{GFP}^{+}$neutrophils. As shown in Figure 5A, at the end of the treatments, all treated groups had statistically significantly fewer GFP+WBC and neutrophils compared with the control group $(P<0.001)$, and the combination was statistically significantly more effective than imatinib $(P<0.01$ for both WBC and neutrophils) or DS-5272 alone $(P<0.01$ for WBC and $P=0.0264$ for neutrophils; left panel). At 7 weeks after treatments, only the combination group demonstrated statistically significant decreases in $\mathrm{GFP}^{+} W B C(P=0.0134)$ and $\mathrm{GFP}^{+}$neutrophils $(P=0.0139)$ compared with the control group (middle panel). At 11 weeks after treatments, although all treatment groups still showed lower leukemia burden compared with controls, no statistical significance was reached (right panel). However, only $50 \%$ (7 of 14 ) of mice in the control group, compared to $60 \%$ (6 of 10 ) in the imatinib-treated group, $67 \%$ (10 of 15) in the DS-5272-treated group, and 71\% (10 of 14 ) in the combination treatment group, remained alive at the end of treatment.

Treatments greatly improved overall survival in CMLbearing mice $(P=0.0066)$ (Figure $5 \mathrm{~B})$. Although not statistically significant, the median overall survival for the DS5272 or imatinib group was 211 and 249 days, respectively; both were markedly longer than that of the untreated control group (141 days). The mice treated with the combination had a median overall survival of 326 days, which was statistically significantly longer than that of the control $(P=0.001)$, the imatinib-treated $(P=0.039)$, and the DS-5272treated $(P=0.047)$ groups. The mice seemed to tolerate the treatments well. No obvious weight loss was observed in the treated mice (Online Supplementary Figure S2).

These data indicated that, like imatinib, inhibition of MDM2 by DS-5272 also had anti-leukemia activity in $\mathrm{CML}$, and the combination had significantly more than each agent alone. The mice treated with the combination had deeper and longer lasting responses, suggesting that combined inhibition of MDM2 and BCR-ABL1 may target CML stem cells.

\section{Combined inhibition of MDM2 and BCR-ABL1 targets chronic myeloid leukemia stem cells in BCR-ABL1 transgenic mice}

To determine whether combined inhibition of MDM2 and BCR-ABL1 targeted CML stem cells, we carried out another set of experiments (see Figure 4A). At the end of treatments, BM cells were obtained from each group for the secondary transplantation. Unfortunately, we did not obtain sufficient cells from the DS-5272 treatment group and the secondary transplantation was conducted using only cells from the untreated control and imatinib or imatinib/DS-5272 treated mice. The number of mice with evidence of engraftment in $\mathrm{PB}$ at 16 weeks was determined by flow cytometry. Engraftment was defined as GFP levels $>1 \%$ (above the background levels in the $\mathrm{PB}$ of $\mathrm{FVB} / \mathrm{N}$ mice without cell injection).

Figure 6A shows the percentage of $\mathrm{GFP}^{+}$cells in $\mathrm{PB}$ samples of mice injected with various amounts of cells from mice treated with different agents. Based on the engrafted and total mice transplanted in each group, we calculated the leukemia LT-HSC frequency. The CML LT-HSC frequency was statistically significantly different among the groups $(P=0.0039)$ (Figure 6A), and statistically significantly decreased by the combination treatment compared with imatinib alone $(P=0.0131)$ or the untreated control $(P=0.0012)$. No statistically significant difference was observed between imatinib-treated and untreated groups $(P=0.38)$, as expected. For every LT-HSC detected in the combination-treated group, three times as many cells were detected in the imatinib group, and four times as many cells were detected in the control group.

We also collected BM cells from mice that received $0.25 \times 10^{6}$ cells/mouse and determined GFP positivity by flow cytometry and BCR-ABL1 RNA levels by RT-PCR. As shown in Figure $6 \mathrm{~B}$, engraftment rates in $\mathrm{BM}$ were similar to those in PB samples (Figure 6A). The number of mice showing markedly lower BM BCR-ABL1 RNA levels (3 logs lower than that in controls) was consistent with the number of mice with GFP $<1 \%$ in both $\mathrm{BM}$ and $\mathrm{PB}$ samples (Figure 6B).

\section{Discussion}

Effectively treating patients with blast crisis CML, and eradicating CML stem cells to achieve cures and prevent disease progression to blast crisis, are two major challenges facing CML therapy. We previously reported that combination of TKI with a MDM2 inhibitor enhanced apoptosis induction in not only proliferating but also quiescent blast crisis CML progenitor cells in vitro. ${ }^{27}$ In the current study, we investigated if this combination strategy has the potential to target CML stem cells and improve cure rates in CML-CP.

Using an inducible transgenic Scl-tTa-BCR-ABL1 mouse model, we demonstrated that CML and CML stem/progenitor cells have increased p53 signaling and are more sensitive to $\mathrm{BH} 3$ peptide-induced apoptosis. This increase was further validated in samples obtained from patients with newly-diagnosed CML compared with normal BM controls, indicating oncogenic induction of p53. This observation supports the idea that CML cells have a propensity to death induction, and activation of p53 by MDM2 inhibition sensitizes CML to TKI. Indeed, we demonstrated that combined inhibition of MDM2 and BCR-ABL1 activated p53 signaling, targeted CML stem/progenitor cells, prolonged mouse survival, and decreased CML LT-HSC.

In contrast to the report by Abraham et al. ${ }^{24}$ we found 
increased levels of p53 and several of its targets in CML BM cells compared to those from normal controls, in both mouse models and human samples. These findings are consistent with a previous report that the BCR-ABL1 fusion protein in CML cells promotes p53 accumulation, but antagonizes its activity by modulating the p53-MDM2 regulatory loop. ${ }^{33}$ A recent study also showed that $\mathrm{CD} 34^{+}$ progenitor cells from CML-CP patients expressed statistically significantly higher phosphorylated p53 (Ser15) compared to $\mathrm{CD}_{34}{ }^{+}$cells from health donors. ${ }^{34}$

We found that pro-apoptotic BAX and NOXA were increased in CML cells compared with normal controls. BCR-ABL1 is known to up-regulate anti-apoptotic proteins such as MCL-1 and BCL-XL to support CML cell survival. We previously reported higher anti-apoptotic BCL-2 levels in CML cells and LSK cells in the same mouse model ${ }^{20}$ These anti-apoptotic BCL-2 proteins likely antagonize the pro-apoptotic BCL-2 proteins tilting the balance towards cell survival, which makes CML cells more dependent on anti-apoptotic BCL-2 proteins. This notion is consistent with our results showing that $\mathrm{BM} \mathrm{CD} 45^{+}$cells and LSK cells from the CML mice were more sensitive to $\mathrm{BH} 3$ peptide-induced apoptosis than those cells from the control mice. Furthermore, it was previously reported that, although imatinib does not directly affect p53 levels, it abrogated nutlin-3-induced p21, ${ }^{35}$ which is known to block the cell cycle and suppress apoptosis. ${ }^{36}$

We were able to detect increased p53, NOXA, and BAX in CML LSK cells from mouse BM treated with DS-5272, or the combination, 24 hours after treatments. The short in vivo half-life of DS-5272 may contribute to the diminished induction of p53 and its target proteins. It is important to point out that although TKI are inactive against CML stem cells, they do inhibit BCR-ABL1 activity in these cells. ${ }^{6}$ The balance of pro- and anti-apoptotic proteins decides cell death or survival. Activation of p53induced apoptotic signaling by MDM2 inhibition together with inhibition of BCR-ABL1-regulated survival pathway by TKI likely push CML cells/stem cells towards death. This is supported by our previous study in blast crisis CML demonstrating that nutlin3a induced p53 and proapoptotic proteins PUMA and BAX, while nilotinib suppressed BCR-ABL1 signaling and decreased anti-apoptotic proteins BCL-XL and MCL-1, and that their combination synergistically induced cell death even in blast crisis CML cells resistance to TKI. ${ }^{27}$ Wendel et al. reported that loss of p53 hampers the anti-leukemia response to BCR-ABL inhibition in a BCR-ABL1 transgenic mouse model, ${ }^{37}$ suggesting that activation of p53 signaling may enhance TKI activities in CML.

You et al. recently demonstrated that JNJ-26854165, another MDM2 inhibitor, is active in CML cells through promoting BCR-ABL proteosomal degradation, independent of $\mathrm{p} 53{ }^{38}$ This is not surprising since several reports have shown p53-independent anticancer activity of JNJ26854165. ${ }^{39-41}$ DS-5272, derived from a candidate MDM2 inhibitor by chemical modifications to improve its potency and physicochemical property, is a highly selective and potent MDM2 inhibitor. Al Although we cannot state that DS-5272 works entirely in a p53-dependent manner, espe- cially since $\mathrm{MDM} 2$ has functions other than antagonizing p53, its use alone or in combination with a TKI increased p53, NOXA, and BAX, suggesting that it functions, at least in part, through increasing the p53 signaling.

Tyrosine kinase inhibitors have been proven to be highly effective in controlling CML, but in most cases they do not cure the disease. Although imatinib significantly deceased CML LSK cells in BM at the end of the treatment in the transgenic mouse model, the combination was more effective. Imatinib had no effect on spleen leukemia LSK. The different effects of imatinib on BM and spleen CML LSK is not clear and it may involve microenvironmental factors. However, the combination also significantly decreased spleen CML LSK cells. Importantly, imatinib by itself did not significantly reduce leukemia LTHSC frequency in second transplantation, but rather did so only when combined with DS-5272. Similarly, nilotinib itself also did not decrease leukemia LT-HSC frequency in a second transplantation, as shown in our previous study. ${ }^{20}$

The mechanism of BCR-ABL1-driven p53 activation is not fully understood. However, BCR-ABL1-mediated hyper-proliferative signals likely contribute to the activation. The combination of MDM2 inhibition and TKI profoundly prolonged overall survival in our mouse model. In addition to modulating apoptosis regulators, other pathways may also be involved. For example, it was reported that TKI nilotinib inhibits MDM2 and induces a p53-independent apoptosis by down-regulating XIAP. ${ }^{43}$ Kojima et al. $^{44}$ showed that inhibition of MDM2 with nutlin decreased CXCL12 in stromal cells, a critical component of the BM microenvironment that supports leukemia-BM microenvironment interactions and confers drug resistance. Furthermore, whether mouse immunity is regulated by the combination treatment is unknown, which warrants future investigation.

Data from this study, together with our previous report in blast crisis $\mathrm{CML}^{27}$ demonstrate that combined inhibition of MDM2 and BCR-ABL1 tyrosine kinase can target CML cells and CML stem/progenitor cells, and it has the potential to overcome TKI resistance and significantly improve outcomes in CML. Furthermore, we have demonstrated that BCL-2 is a key survival factor of CML stem cells, and targeting BCL-2, combined with a TKI, had the potential to eradicate CML stem cells. ${ }^{20}$ Adding a MDM2 inhibitor, which activates p53 and induces proapoptotic BCL-2 proteins to the combination, will likely further improve the therapeutic potential for patients with CML, which certainly warrants future clinical investigations.

\section{Funding}

The authors would like to thank the research funding from Daiichi Sankyo (to BZC) and by National Institutes of Health grants P01CA49639 and P30CA016672 and the Paul and Mary Haas Chair in Genetics (to MA) and by NCI-SBIR grant 2R44CA203610-02A1 (to Eutropics) for supporting this study.

\section{Acknowledgments}

Erica Goodoff from Scientific Publication at MD Anderson Cancer Center and Numsen Hail, Jr. for editing and assisting with the manuscript preparation. 


\section{References}

1. Heisterkamp N, Stephenson JR, Groffen J, et al. Localization of the c-ab1 oncogene adjacent to a translocation break point in chronic myelocytic leukaemia. Nature. 1983;306(5940):239-242.

2. Nowell PC, Hungerford DA. Chromosome studies in human leukemia. II. Chronic granulocytic leukemia. J Natl Cancer Inst. 1961;27:1013-1035.

3. Rowley JD. Letter: A new consistent chromosomal abnormality in chronic myelogenous leukaemia identified by quinacrine fluorescence and Giemsa staining. Nature. 1973;243(5405):290-293

4. Holyoake T, Jiang X, Eaves C, Eaves A. Isolation of a highly quiescent subpopulation of primitive leukemic cells in chronic myeloid leukemia. Blood. 1999;94(6):20562064.

5. Copland M, Hamilton A, Elrick LJ, et al. Dasatinib (BMS-354825) targets an earlier progenitor population than imatinib in primary CML but does not eliminate the quiescent fraction. Blood. 2006;107(11):45324539.

6. Corbin AS, Agarwal A, Loriaux M, Cortes J, Deininger MW, Druker BJ. Human chronic myeloid leukemia stem cells are insensitive to imatinib despite inhibition of BCR-ABL activity. J Clin Invest. 2011;121(1):396-409.

7. Graham SM, Jorgensen HG, Allan E, et al. Primitive, quiescent, Philadelphia-positive stem cells from patients with chronic myeloid leukemia are insensitive to STI571 in vitro. Blood. 2002:99(1):319-325.

8. Mahon FX, Rea D, Guilhot J, et al. Discontinuation of imatinib in patients with chronic myeloid leukaemia who have maintained complete molecular remission for at least 2 years: the prospective, multicentre Stop Imatinib (STIM) trial. Lancet Oncol. 2010;11(11):1029-1035

9. Rea D, Nicolini FE, Tulliez M, et al. Discontinuation of dasatinib or nilotinib in chronic myeloid leukemia: interim analysis of the STOP 2G-TKI study. Blood. 2017;129(7):846-854

10. Ross DM, Branford S, Seymour JF, et al. Safety and efficacy of imatinib cessation for CML patients with stable undetectable minimal residual disease: results from the TWISTER study. Blood. 2013;122(4):515522 .

11. Saussele S, Richter J, Hochhaus A, Mahon FX. The concept of treatment-free remission in chronic myeloid leukemia. Leukemia. 2016;30(8):1638-1647.

12. Carter BZ, Andreeff M. Eradication of CML stem cells. Oncoscience. 2016;3(11-12):313315.

13. Hamad A, Sahli Z, El Sabban M, Mouteirik M, Nasr R. Emerging therapeutic strategies for targeting chronic myeloid leukemia stem cells. Stem Cells Int. 2013;2013: 724360 .

14. Holyoake TL, Vetrie D. The chronic myeloid leukemia stem cell: stemming the tide of persistence. Blood. 2017;129(12): 1595-1606.

15. Zhang B, Strauss AC, Chu S, et al. Effective targeting of quiescent chronic myelogenous leukemia stem cells by histone deacetylase inhibitors in combination with imatinib mesylate. Cancer Cell. 2010;17(5):427-442.
16. Zhou H, Mak PY, Mu H, et al. Combined inhibition of beta-catenin and Bcr-Abl synergistically targets tyrosine kinase inhibitorresistant blast crisis chronic myeloid leukemia blasts and progenitors in vitro and in vivo. Leukemia. 2017;31 (10):2065-2074.

17. Goff DJ, Court Recart A, Sadarangani A, et al. A Pan-BCL2 inhibitor renders bone-marrow-resident human leukemia stem cells sensitive to tyrosine kinase inhibition. Cell Stem Cell. 2013;12(3):316-328.

18. Kuroda J, Kimura S, Strasser A, et al. Apoptosis-based dual molecular targeting by INNO-406, a second-generation Bcr-Ab inhibitor, and $\mathrm{ABT}-737$, an inhibitor of antiapoptotic Bcl-2 proteins, against Bcr-Ablpositive leukemia. Cell Death Differ. 2007;14(9):1667-1677.

19. Mak DH, Wang RY, Schober WD, et al. Activation of apoptosis signaling eliminates CD34+ progenitor cells in blast crisis CML independent of response to tyrosine kinase inhibitors. Leukemia. 2012;26(4):788-794.

20. Carter BZ, Mak PY, Mu H, et al. Combined targeting of BCL-2 and BCR-ABL tyrosine kinase eradicates chronic myeloid leukemia stem cells. Sci Transl Med. 2016;8(355):355ra117.

21. Miyashita T, Reed JC. Tumor suppressor p53 is a direct transcriptional activator of the human bax gene. Cell. 1995;80(2):293299

22. Sax JK, Fei P, Murphy ME, Bernhard E, Korsmeyer SJ, El-Deiry WS. BID regulation by $\mathrm{p} 53$ contributes to chemosensitivity. Nat Cell Biol. 2002;4(11):842-849.

23. Yu J, Wang Z, Kinzler KW, Vogelstein B, Zhang L. PUMA mediates the apoptotic response to p53 in colorectal cancer cells. Proc Natl Acad Sci U S A. 2003;100(4):19311936.

24. Abraham SA, Hopcroft LE, Carrick E, et al Dual targeting of p53 and c-MYC selectivey eliminates leukaemic stem cells. Nature. 2016;534(7607):341-346.

25. Li L, Wang L, Li L, et al. Activation of p53 by SIRT1 inhibition enhances elimination of CML leukemia stem cells in combination with imatinib. Cancer Cell. 2012;21(2):266281.

26. Peterson LF, Mitrikeska E, Giannola D, et al. p53 stabilization induces apoptosis in chronic myeloid leukemia blast crisis cells. Leukemia. 2011;25(5):761-769.

27. Carter BZ, Mak PY, Mak DH, et al. Synergistic effects of p53 activation via MDM2 inhibition in combination with inhibition of Bcl-2 or Bcr-Abl in CD34+ proliferating and quiescent chronic myeloid leukemia blast crisis cells. Oncotarget. 2015;6(31):30487-30499.

28. Huettner CS, Koschmieder S, Iwasaki H, et al. Inducible expression of $\mathrm{BCR} / \mathrm{ABL}$ using human CD34 regulatory elements results in a megakaryocytic myeloproliferative syndrome. Blood. 2003;102(9):3363-3370.

29. Koschmieder S, Gottgens B, Zhang P, et al. Inducible chronic phase of myeloid leukemia with expansion of hematopoietic stem cells in a transgenic model of BCRABL leukemogenesis. Blood. 2005;105(1): 324-334

30. Han L, Qiu P, Zeng Z, et al. Single-cell mass cytometry reveals intracellular survival/proliferative signaling in FLT3ITD-mutated AML stem/progenitor cells.
Cytometry A. 2015;87(4):346-356.

31. Ishizawa J, Kojima $K, M c Q u e e n ~ T$, et al Mitochondrial Profiling of Acute Myeloid Leukemia in the Assessment of Response to Apoptosis Modulating Drugs. PLoS One. 2015;10(9):e0138377.

32. Hu Z, Pan XF, Wu FQ, et al. Synergy between proteasome inhibitors and imatinib mesylate in chronic myeloid leukemia. PLoS One. 2009:4(7):e6257.

33. Levav-Cohen Y, Goldberg Z, Zuckerman V, Grossman T, Haupt S, Haupt Y. C-Abl as a modulator of p53. Biochem Biophys Res Commun. 2005;331(3):737-749.

34. Ricciardi MR, Salvestrini V, Licchetta R, et al. Differential proteomic profile of leukemic CD34+ progenitor cells from chronic myeloid leukemia patients. Oncotarget. 2018;9(31):21758-21769.

35. Kurosu T, Wu N, Oshikawa G, Kagechika $\mathrm{H}$, Miura $\mathrm{O}$. Enhancement of imatinibinduced apoptosis of BCR/ABL-expressing cells by nutlin-3 through synergistic activation of the mitochondrial apoptotic pathway. Apoptosis. 2010;15(5):608-620.

36. Carter BZ, Mak DH, Schober WD, et al Simultaneous activation of p53 and inhibition of XIAP enhance the activation of apoptosis signaling pathways in AML. Blood. 2010;115(2):306-314

37. Wendel HG, de Stanchina E, Cepero E, et al. Loss of p53 impedes the antileukemic response to BCR-ABL inhibition. Proc Nat Acad Sci U S A. 2006;103(19):7444-7449.

38. You L, Liu H, Huang J, et al. The novel anticancer agent JNJ-26854165 is active in chronic myeloid leukemic cells with unmutated BCR/ABL and T315I mutan $\mathrm{BCR} / \mathrm{ABL}$ through promoting proteosoma degradation of $\mathrm{BCR} / \mathrm{ABL}$ proteins. Oncotarget 2017:8(5):7777-7790

39. Jones RJ, Gu D, Bjorklund CC, et al. The novel anticancer agent JNJ-26854165 induces cell death through inhibition of cholesterol transport and degradation of ABCA1. I Pharmacol Exp Ther 2013:346(3):381-392.

40. Kojima K, Burks JK, Arts J, Andreeff M. The novel tryptamine derivative JNJ-26854165 induces wild-type p53- and E2F1-mediated apoptosis in acute myeloid and lymphoid leukemias. Mol Cancer Ther. 2010;9(9): 2545-2557.

41. Lehman JA, Hauck PM, Gendron JM, et al. Serdemetan antagonizes the $\mathrm{Mdm} 2$ HIF1alpha axis leading to decreased levels of glycolytic enzymes. PLoS One. 2013;8(9):e74741.

42. Miyazaki M, Uoto K, Sugimoto Y, et al Discovery of DS-5272 as a promising can didate: A potent and orally active p53MDM2 interaction inhibitor. Bioorg Med Chem. 2015;23(10):2360-2367.

43. Zhang H, Gu L, Liu T, Chiang KY, Zhou M Inhibition of MDM2 by nilotinib contributes to cytotoxicity in both Philadelphia-positive and negative acute lymphoblastic leukemia. PLoS One. 2014;9(6):e100960.

44. Kojima K, McQueen T, Chen Y, et al. p53 activation of mesenchymal stromal cells partially abrogates microenvironmentmediated resistance to FLT3 inhibition in AML through HIF-1alpha-mediated downregulation of CXCL12. Blood. 2011, 118(16):4431-4439 